\title{
Invited Discussion on: Autologous Fat Grafting with Percutaneous Fasciotomy and Reduction of the Nipple-Areolar Complex for the Correction of Tuberous Breast Deformity in Teenagers
}

\author{
Ozan Sozer $^{1} \cdot$ Paul Phillips $^{1}$
}

Received: 2 December 2019/Accepted: 3 December 2019/Published online: 12 December 2019

(C) Springer Science+Business Media, LLC, part of Springer Nature and International Society of Aesthetic Plastic Surgery 2019

Level of Evidence $V$ This journal requires that authors assign a level of evidence to each article. For a full description of these Evidence-Based Medicine ratings, please refer to the Table of Contents or the online Instructions to Authors www.springer.com/00266.

The authors present their technique of fat grafting and nipple-areolar complex reduction for correction of tuberous breasts in the adolescent patient. The described technique has the benefit of avoiding the use of implants and reconstructive flaps or excisional approaches which although common practice, may have lasting effects in the young, teenage population. Many corrective techniques have been previously described depending on the degree of tuberous deformity [1]. When performing these reconstructive procedures, the paucity of abdominal tissue does limit the availability of several flap options (DIEP and SIEA) [2]. The author's described technique is able to correct the deformity with minimal (hematoma, seroma, etc.) complications; however, long-term cancer surveillance follow-up implications are still relatively unknown. We commend the authors on their excellent results and lack of complications. We agree the usage of implants and autologous flaps should be avoided in adolescent patients to preserve the ability of breastfeeding, nipple function/ sensation and avoid donor site morbidity [3].

The age of intervention appears to be early despite the patient's achievement of Tanner stage $\mathrm{V}$ as the average age was 14.9 years, whereas the breast size may continue to grow for several years. In our practice, if a patient in this age-group desires tuberous breast correction, we initially

Ozan Sozer

ozansozer@gmail.com; doctor@elpasoplasticsurgery.com

1 El Paso Cosmetic Plastic Surgery Center, El Paso, TX, USA focus on the four primary structural characteristics of tuberous breasts [4] - narrow breast base width (BBW), underdeveloped lower pole, short nipple to IMF distance, and herniation of the breast parenchyma through the areola of the tuberous breast deformity. We would like to limit the amount of fat transferred in the adolescent period. Our goal is correcting the tubular deformity with minimal changes in volume. Choi et al. [5] demonstrated that larger volumes (mean $151 \mathrm{~mL}$ ) of fat transferred had higher rates of retention $(52.3 \%)$ at 140 days when compared to those with smaller volumes (51 mL and $27.1 \%$ retention). Thus, larger amounts of grafted fat may predispose patients to large breast volume changes if the patient has weight fluctuations as they age. As opposed to the author's average of $220 \mathrm{~mL}$ per breast, we prefer to limit the volume of fat transferred to smaller amounts (approximately $150 \mathrm{~mL}$ per breast), which adequately expands the breast footprint both horizontally and inferiorly. By focusing on the structural footprint of the breast, the distance between the nipple and IMF is lengthened and the BBW is also widened.

Herniation of the breast tissue through the areola is relieved by a circumareolar skin reduction, in a similar fashion to the author's described methods. We concur with the author's advisement to anticipate extensive ecchymosis of the breasts and donor sites for several days postoperatively. Development of the inferior pole can be accomplished by using percutaneous rigottomies and fat grafting to the lower region of the breast. As opposed to the author's use of a "pickle-fork" cannula, we prefer using an 18-gauge needle. We believe an 18-gauge needle allows us to perform rigottomies in a sweeping motion rather than in a linear motion as described. By using a sweeping motion, we feel the recipient matrix can be better prepared for expansion.

Once the structural deformities are corrected and the patient reaches an age older than 18 , further improvement 
can be achieved by utilizing breast implants or further fat grafting after discussing pros and cons of each procedure with the patient. Upon review of the presented article, it is noted the patients did express a desire for additional volume. This desire is often alleviated by placement of implants of a size decided preoperatively through imaging devices such as Vectra or implant sizing systems simulating the postoperative outcome. The underdeveloped inferior pole is augmented, and the base width of the breast is increased by selecting implants slightly wider than the native BBW. A dual-plane dissection approach is used to allow the incised margin of the pectoralis muscle to shift superiorly and allow the implant to expand the lower pole of the breast [6]. If a double-bubble deformity develops following the use of breast implants, additional fat grafting can be performed to alleviate the irregularity [7].

As discussed in the article, a more validated questionnaire such as the BREAST-Q would be appropriate to establish the effectiveness of patient satisfaction after undergoing the techniques presented by the author.

\section{Compliance with Ethical Standards}

Conflict of interest The authors declare that they have no conflicts of interest to disclose.

Ethical Approval This article does not contain any studies with human participants or animals performed by any of the authors.
Informed Consent For this type of study, informed consent is not required.

\section{References}

1. Latham K, Fernandez S, Iteld L et al (2006) Pediatric breast deformity. J Craniofac Surg 17:454

2. Gautam AK, Allen RJ Jr, LoTempio MM et al (2007) Congenital breast deformity reconstruction using perforator flaps. Ann Plast Surg 58:353

3. Dupéré S, Bergeron L, Bortoluzzi P, Del-Duca T, CaouetteLaberge L (2007) Donor-site morbidity of the inferior gluteal musculocutaneous flap for breast reconstruction in teenagers. Ann Plast Surg 59:617

4. Winocour S, Lemaine V (2013) Hypoplastic breast anomalies in the female adolescent breast. Semin Plast Surg 27:42

5. Choi M, Small K, Levovitz C et al (2013) The volumetric analysis of fat graft survival in breast reconstruction. Plast Reconstr Surg 131:185

6. Kolker A, Collins M (2015) Tuberous breast deformity: classification and treatment strategy for improving consistency in aesthetic correction. Plast Reconstr Surg 135:73

7. Bresnick S (2016) Management of a common breast augmentation complication. Ann Plast Surg 76:18

Publisher's Note Springer Nature remains neutral with regard to jurisdictional claims in published maps and institutional affiliations. 